\title{
A higher version of Zappa products for monoids
}

\author{
Ahmet Sinan Cevik*1,3 (D), Suha Ahmad Wazzan ${ }^{1}$ (D), Firat Ates $^{2}$ (D) \\ ${ }^{1}$ Department of Mathematics, KAU King Abdulaziz University, Science Faculty, 21589, Jeddah-Saudi \\ Arabia \\ ${ }^{2}$ Department of Mathematics, Science and Art Faculty, Balikesir University, Campus, 10100, Balikesir, \\ Turkey \\ ${ }^{3}$ Department of Mathematics, Faculty of Science, Selcuk University, Campus, 42075, Konya, Turkey
}

\begin{abstract}
For arbitrary monoids $A$ and $B$, a presentation for the restricted wreath product of $A$ by $B$ that is known as the semi-direct product of $A^{\oplus B}$ by $B$ has been widely studied. After that a presentation for the Zappa product of $A$ by $B$ was defined which can be thought as the mutual semidirect product of given these two monoids under a homomorphism $\psi: A \rightarrow \mathcal{T}(B)$ and an anti-homomorphism $\delta: B \rightarrow \mathcal{T}(A)$ into the full transformation monoid on $B$, respectively on $A$. As a next step of these above results, by considering the monoids $A^{\oplus B}$ and $B^{\oplus A}$, we first introduce an extended version (generalization) of the Zappa product and then we prove the existence of an implicit presentation for this new product. Furthermore we present some other outcomes of the main theories in terms of finite and infinite cases, and also in terms of groups. At the final part of this paper we point out some possible future problems related to this subject.
\end{abstract}

Mathematics Subject Classification (2020). 20E22, 20F05, 20L05, 20M05

Keywords. Knit products, Wreath products, Zappa products, presentations

\section{Introduction}

Study on the product of groups have received much attention in the literature. During these studies, people investigated this group product which is constructed by subgroups either in terms of permutability (cf. $[6,9,17]$ ) or in terms of an extension (cf. [5, 24]). Nevertheless, direct, semidirect and (standard) wreath products are the most famous structures among these extension constructions (see, for instance, [10,14, 18, 20, 25]). As a next step of these products, some other people also studied Zappa (or Zappa-Szép) products $([13,16,27,28])$ which is also referred as bilateral semidirect products $([22])$, general products $([23])$ or knit products $([1,26])$. Unlikely semi-direct products, none of the factor is normal in the Zappa product of any two groups. In other words, for a group $G$ with subgroups $A$ and $B$ that satisfy $A \cap B=\left\{1_{G}\right\}$ and $G=A B$, we know that each element $g \in G$ is expressible (uniquely) as $g=a b$ with $a \in A$ and $b \in B$. Now to reserve

\footnotetext{
*Corresponding Author.

Email addresses: ahmetsinancevik@gmail.com (A.S. Cevik), swazzan@kau.edu.sa (S.A. Wazzan), firat@balikesir.edu.tr (F. Ates)

Received: 13.03.2020; Accepted: 31.05.2020
} 
certain products, let us consider an element $b a \in G$. In fact there must be unique elements $b^{\prime} \in B$ and $a^{\prime} \in A$ such that $b a=a^{\prime} b^{\prime}$. This actually implies two functions

$$
(b, a) \longmapsto b^{a} \in B, \quad(b, a) \longmapsto b \cdot a={ }^{b} a \in A
$$

which are unique and so satisfy

$$
b a=(b . a)\left(b^{a}\right)={ }^{b} a b^{a},
$$

for all $b \in B$ and $a \in A$.

According to the references $[13,22,23,25]$, by considering the action given (1.1), the monoid version of the Zappa product of any two monoids can be defined as follows.

For any two monoids $A$ and $B$, let us consider a homomorphism $\psi: A \rightarrow \mathcal{T}(B)$ and an anti-homomorphism $\delta: B \rightarrow \mathcal{T}(A)$ such that $\mathcal{T}($.$) denotes the full transformation monoid.$ For $a \in A, b \in B$, denote the operation of $(a) \psi$ on $B$ by $b \longmapsto(a) \psi=b^{a}$ and the operation of $(b) \delta$ on $A$ by $a \longmapsto(a) \delta_{b}={ }^{b} a$. For every elements $a, a_{1}, a_{2} \in A, b, b_{1}, b_{2} \in B$, suppose that the conditions

$$
\begin{aligned}
& b^{1_{A}}=b, \quad 1_{B}^{a}=1_{B}, \quad\left(1_{A}\right) \delta_{b}=1_{A}, \quad(a) \delta_{1_{B}}=a, \\
& b^{\left(a_{1} a_{2}\right)}=\left(b^{a_{1}}\right)^{a_{2}}, \quad(a) \delta_{b_{1} b_{2}}=\left((a) \delta_{b_{2}}\right) \delta_{b_{1}}, \\
& \left(b_{1} b_{2}\right)^{a}=b_{1}^{(a) \delta_{b_{2}}} b_{2}^{a} \quad \text { and } \quad\left(a_{1} a_{2}\right) \delta_{b}=\left(a_{1}\right) \delta_{b}\left(a_{2}\right) \delta_{b^{a_{1}}}
\end{aligned}
$$

are all true. Then the set $A \times B$ defines the Zappa product $A_{\delta} \times{ }_{\psi} B$ (cf. [13,22]) of $A$ and $B$ which is of course a monoid with respect to the multiplication,

$$
\left(a_{1}, b_{1}\right)\left(a_{2}, b_{2}\right)=\left(a_{1}\left(a_{2}\right) \delta_{b_{1}}, b_{1}^{a_{2}} b_{2}\right) .
$$

Assume that $A$ has a monoid presentation $\mathcal{P}_{A}=[X ; R]$ while $B$ has $\mathcal{P}_{B}=[Y ; S]$. Then, by [23, Theorem 2], a presentation for $A_{\delta} \times{ }_{\psi} B$ with the structure defined by (1.3) on the set $A \times B$ is given as $\mathcal{P}=[X, Y ; R, S, T]$ in which the relator $T$ consists of all ordered elements $\left(b a,{ }^{b} a b^{a}\right)$, as given in $(1.2)$, for $(b, a) \in B \times A$.

Since there are some difficulties in the meaning of embedding for the factors in the product unless they are not taken as identities, throughout in this paper we will not attempt to study the cases of Zappa products for semigroups.

To give another preliminary material for the next section, let us recall the fundamentals of standard wreath products of any two monoids $A$ and $B$. First let us consider the monoid $A^{\oplus B}$ which is the direct product of the number of $B$ copies of $A$. In fact $A^{\oplus B}$ can be thought as the set of all functions $f$ having finite support. Suppose that $\psi: A^{\oplus B} \rightarrow \mathcal{T}(B)$ is a homomorphism and $\delta: B \rightarrow \mathcal{T}\left(A^{\oplus B}\right)$ is an anti-homomorphism where $\mathcal{T}($.$) is the$ full transformation monoid on $B$ and $A^{\oplus B}$, respectively, as previously. For $g \in A^{\oplus B}$ and $b \in B$, let us denote the operation of $(g) \psi$ on $B$ by $b \longmapsto b$ and operation of $(b) \delta$ on $A^{\oplus B}$ by $g \longmapsto(g) \delta_{b}={ }^{b} g$. Then the set $A^{\oplus B} \times B$ defines a monoid $A$ \ $B$ (namely the (standard) wreath product of $A$ by $B$ ) with the operation $\left(f, b_{1}\right)\left(g, b_{2}\right)=\left(f^{b_{1}} g, b_{1} b_{2}\right)$, and the identity is $\left(I, 1_{B}\right)$, where $(x) I=1_{A}$ (cf. [14,18,20,22]). It is clear that $A$ ? $B$ is actually the semidirect product of $A^{\oplus B}$ by $B$ and notated by $A^{\oplus B} \times{ }_{\delta} B$. Now, by taking into account the same presentations $\mathcal{P}_{A}$ and $\mathcal{P}_{B}$ for the monoids $A$ and $B$ as in above, for each $b \in B$, let us assume the set $X_{b}=\left\{x_{b}: x \in X\right\}$ is a copy of $X$ and the set $R_{b}$ is the corresponding copy of $R$. So, for $x, x^{\prime} \in X, y \in Y, b, e \in B, b \neq e$, the monoid $A$ 々 $B$ has a presentation

$$
\left[X_{b}, Y ; R_{b}, S, x_{b} x_{e}^{\prime}=x_{e}^{\prime} x_{b}, y x_{b}=\left(\prod_{m \in b y^{-1}} x_{m}\right) y\right]
$$

(cf. $[2,14,18,25]$ ). 


\section{A higher version of the Zappa product}

By combining the definitions of Zappa and (standard) wreath products, the main purposes of this section are to define and study a generalized version of the Zappa product of $A^{\oplus B}$ by $B^{\oplus A}$, namely restricted generalized Zappa product $A^{\oplus B}{ }_{\delta} \times{ }_{\psi} B^{\oplus A}$ with an operation adapted from (1.3). Additionally, by considering the presentation in (1.4), we will prove the existence of an implicit presentation for this product (see Theorem 2.2 below). Moreover, by taking into account a special case $A^{\oplus B}{ }_{\delta} \times{ }_{\psi} B$ of this new product, we will state and prove some consequences of this theorem.

Let $A$ and $B$ be monoids, and let the set $A^{\times B}$ denotes the Cartesian product of the number of $B$ copies of the monoid $A$ while the set $A^{\oplus B}$ denotes the corresponding direct product as in wreath products. Recall that $A^{\oplus B}$ can be thought as the set of whole functions $f$ with finite support (in other words, functions with the property $(x) f=1_{A}$ for all but finitely many $x$ in $B$ ). Hence a generalization of restricted and unrestricted Zappa products of the monoid $A^{\oplus B}$ by the monoid $B^{\oplus A}$ are defined on $A^{\times B} \times B^{\times A}$ and $A^{\oplus B} \times B^{\oplus A}$, respectively, with the multiplication

$$
(f, h)(g, k)=\left(f(g) \delta_{h},(h) \psi_{g} k\right)=\left(f^{h} g, h^{g} k\right),
$$

where $\delta: B^{\oplus A} \rightarrow \mathcal{T}\left(A^{\oplus B}\right),(g) \delta_{h}={ }^{h} g$ and $\psi: A^{\oplus B} \rightarrow \mathcal{T}\left(B^{\oplus A}\right),(h) \psi_{g}=h^{g}$ are defined by, for $a \in A$ and $b \in B$,

$$
{ }^{h} g={ }^{\left(h^{a}\right)} g \quad \text { and } \quad h^{g}=h^{(b g)} .
$$

Also, for $x \in A$ and $y \in B$, we define

$$
(x) h^{a}=(a x) h \quad \text { and } \quad(y)^{b} g=(y b) g
$$

such that, for all $d \in B, c \in A$,

$$
(d){ }^{\left(h^{a}\right)} g=\left(d h^{a}\right) g \quad \text { and } \quad(c) h^{\left({ }^{b} g\right)}=\left({ }^{b} g c\right) h .
$$

Both these restricted and unrestricted generalized Zappa products are monoids under the multiplication defined in (2.1) with the identity $(\overline{1}, \tilde{1})$, where $\overline{1}: B \rightarrow A,(b) \overline{1}=1_{A}$ and $\tilde{1}: A \rightarrow B,(a) \tilde{1}=1_{B}$, for all $a \in A$ and $b \in B$.

Throughout this paper all generalized Zappa products will be assumed to be restricted and so we will use the notation $A^{\oplus B}{ }_{\delta} \times{ }_{\psi} B^{\oplus A}$ for it. It is clear that the sets $\{(f, \tilde{1}): f \in$ $\left.A^{\oplus B}\right\}$ and $\left\{(\overline{1}, k): k \in B^{\oplus A}\right\}$ are the submonoids of $A^{\oplus B}{ }_{\delta} \times{ }_{\psi} B^{\oplus A}$ which are isomorphic to $A^{\oplus B}$ and $B^{\oplus A}$, respectively. Moreover, for $f \in A^{\oplus B}$ and $k \in B^{\oplus A}$, we definitely have $(f, \tilde{1})(\overline{1}, k)=(f, k)$.

For $a \in A$ and $b \in B$, we now define $\overline{a_{b}}: B \rightarrow A$ and $\widetilde{b_{a}}: A \rightarrow B$ as

$$
(m) \overline{a_{b}}=\left\{\begin{array}{ll}
a, & b=m \\
1_{A}, & \text { otherwise }
\end{array} \quad \text { and } \quad(n) \tilde{b_{a}}=\left\{\begin{array}{ll}
b, & a=n \\
1_{B}, & \text { otherwise }
\end{array} .\right.\right.
$$

Notice that if $f: B \rightarrow A$ and $k: A \rightarrow B$ have finite supports, then

$$
f=\prod_{b \in B} \overline{((b) f)_{b}} \quad \text { and } \quad k=\prod_{a \in A} \widetilde{((a) k)_{a}} .
$$

Also notice that if the monoid $A$ is generated by a set $X$ (so that every $a$ in $A$ is expressible as a finite product $x_{1} x_{2} \cdots x_{n}$ of elements of $X$ ) and if the monoid $B$ is generated by $Y$ (so every $b$ in $B$ is expressible as a finite product $y_{1} y_{2} \cdots y_{m}$ ), then

$$
\overline{a_{b}}=\overline{x_{1_{b}}} \overline{x_{2_{b}}} \cdots \overline{x_{n_{b}}} \quad \text { and } \quad \widetilde{b_{a}}=\widetilde{y_{1_{a}}} \widetilde{y_{2_{a}}} \cdots \widetilde{y_{m_{a}}} \text {. }
$$

After all, we have the following lemma which is actually a generalization of [18, Lemma 2.1]. 
Lemma 2.1. Assume that the sets $X$ and $Y$ generate the monoids $A$ and $B$, respectively. Further, let $\overline{X_{b}}=\left\{\left(\overline{x_{b}}, \tilde{1}\right): b \in B, x \in X\right\}$ and $\widetilde{Y_{a}}=\left\{\left(\overline{1}, \widetilde{y_{a}}\right): a \in A, y \in Y\right\}$. Then the product $A^{\oplus B} \times_{\psi} B^{\oplus A}$ is generated by the set $\left(\bigcup_{b \in B} \overline{X_{b}}\right) \cup\left(\bigcup_{a \in A} \widetilde{Y_{a}}\right)$.

In general, the generating set given in Lemma 2.1 is the best possible for the monoids $A$ and $B$. If $B$ has an indecomposable identity (in other words, for all $b, c \in B, b c=1_{B} \Rightarrow$ $\left.b=c=1_{B}\right)$, then any generating set of $A^{\oplus B}{ }_{\delta} \times{ }_{\psi} B^{\oplus A}$ must contain elements from the generating set of the submonoid $A^{\oplus B} \cong\left\{(f, \tilde{1}): f \in A^{\oplus B}\right\}$ and, in fact, $\bigcup_{b \in B} \overline{X_{b}}$ is the smallest such a set. One may discuss same arguments for $\bigcup_{a \in A} \widetilde{Y_{a}}$ as well.

For simplicity, we will denote the set $\{m \in B: b=m y\}$ with only $b y^{-1}$ (where $b, y \in B$ ) and will denote the set $\{n \in A: a=x n\}$ with only $x^{-1} a$ (where $a, x \in A$ ).

The following theorem generalizes the result presented in [13].

Theorem 2.2. Suppose that the monoids $A$ and $B$ are presented by $[X ; R]$ and $[Y ; S]$, respectively. For each $b \in B$, let $X_{b}=\left\{x_{b}: x \in X\right\}$ denote a copy of $X$, and let $R_{b}$ denote the corresponding copy of $R$. Similarly, for each $a \in A$, let $Y_{a}=\left\{y_{a}: y \in Y\right\}$ be a copy of $Y$, and let $S_{a}$ be the corresponding copy of $S$. Then the (restricted) generalized Zappa product $A^{\oplus B}{ }_{\delta} \times_{\psi} B^{\oplus A}$ is defined by the generators $\left(\bigcup_{b \in B} X_{b}\right) \cup\left(\bigcup_{a \in A} Y_{a}\right)$ and relations

$$
\begin{gathered}
R_{b}, S_{a}, \quad(a \in A, b \in B) ; \\
x_{b} x_{e}^{\prime}=x_{e}^{\prime} x_{b}, \quad\left(x, x^{\prime} \in X, b, e \in B, b \neq e\right) ; \\
y_{a} y_{s}^{\prime}=y_{s}^{\prime} y_{a}, \quad\left(y, y^{\prime} \in Y, a, s \in A, a \neq s\right) ; \\
y_{a} x_{b}=\left(\prod_{m \in b y^{\prime-1}} x_{m}\right)\left(\prod_{n \in x^{\prime-1} a} y_{n}\right)
\end{gathered}
$$

such that the elements $x^{\prime}$ and $y^{\prime}$ in Eq. (2.6) are defined as

$$
x^{\prime}=\prod_{m \in b y^{-1}} x_{m} \quad \text { and } \quad y^{\prime}=\prod_{n \in x^{-1} a} y_{n}
$$

respectively.

Proof. We first recall that, for a set of alphabet $\mathfrak{M}$, the monoid of all words in $\mathfrak{M}$ is notated by $\mathfrak{M}^{*}$.

For $x \in X, b \in B, y \in Y, a \in A$, the mapping $\rho$ from the monoid $\left(\left(\bigcup_{b \in B} X_{b}\right) \cup\left(\bigcup_{a \in A} Y_{a}\right)\right)^{*}$, say $M$, to the product $A^{\oplus B}{ }_{\delta} \times_{\psi} B^{\oplus A}$ defined by $\left(x_{b}\right) \rho=\left(\overline{x_{b}}, \tilde{1}\right)$ and $\left(y_{a}\right) \rho=\left(\overline{1}, \widetilde{y_{a}}\right)$ is surjective as a result of Lemma 2.1. Furthermore, relations in (2.3), (2.4) and (2.5) are all held in $A^{\oplus B}{ }_{\delta} \times{ }_{\psi} B^{\oplus A}$ by the equalities and explanations presented just before Lemma 2.1 .

Now the next step is to obtain relation (2.6). We easily deduce from (2.1) that

$$
\left(\overline{1}, \widetilde{y_{a}}\right)\left(\overline{x_{b}}, \tilde{1}\right)=\left(\widetilde{y_{a}} \overline{x_{b}}, \widetilde{y_{a}} \overline{x_{b}}\right) .
$$

Now by considering (2.2), for each $x \in X$, we can write

$$
\left.\widetilde{y_{a}} \overline{x_{b}}={ }^{\left(\tilde{y}_{a}^{x}\right.}\right)^{x_{b}},
$$

where for $d \in A$,

$$
\begin{aligned}
(d){\widetilde{y_{a}}}^{x}=(x d) \widetilde{y_{a}} & =\left\{\begin{array}{ll}
y, & a=x d \\
1_{B}, & \text { otherwise }
\end{array}= \begin{cases}y, & d \in x^{-1} a \\
1_{B}, & \text { otherwise }\end{cases} \right. \\
& =\prod_{n \in x^{-1} a}(d) \widetilde{y_{n}}=(d) \prod_{n \in x^{-1} a} \widetilde{y_{n}} .
\end{aligned}
$$


So we have $\widetilde{y_{a}}=\prod_{n \in x^{-1} a} \widetilde{y_{n}}$. For simplicity, let us denote $\prod_{n \in x^{-1} a} \widetilde{y_{n}}$ by only $y^{\prime}$. As a result, we obtain

$$
\left.{ }^{\left(\tilde{y}_{a}^{x}\right.}\right)_{\overline{x_{b}}}=y^{\prime} \overline{x_{b}} .
$$

Moreover, for $e \in B$,

$$
\begin{aligned}
& (e)^{y^{\prime} \overline{x_{b}}}=\left(e y^{\prime}\right) \overline{x_{b}}=\left\{\begin{array}{ll}
x, & b=e y^{\prime} \\
1_{A}, & \text { otherwise }
\end{array}= \begin{cases}x, & e \in b y^{\prime-1} \\
1_{A}, & \text { otherwise }\end{cases} \right. \\
& =\prod_{m \in b y^{\prime}-1}(e) \overline{x_{m}}=(e) \prod_{m \in b y^{\prime-1}} \overline{x_{m}} .
\end{aligned}
$$

Therefore ${ }^{y^{\prime}} \overline{x_{b}}=\prod_{m \in b y^{\prime}-1} \overline{x_{m}}$ and finally we have

$$
\left.\widetilde{y_{a}} \overline{x_{b}}={ }^{\left(\tilde{y a}^{x}\right.}\right)^{x_{b}}={ }^{y^{\prime}} \overline{x_{b}}=\prod_{m \in b y^{\prime}-1} \overline{x_{m}} .
$$

Additionally, for each $y \in Y$, by taking into account the second part of (2.2) and its attachments, since

$$
{\widetilde{y_{a}}}^{\overline{x_{b}}}={\widetilde{y_{a}}}^{\left({ }^{y} \overline{x b}_{b}\right)}
$$

we clearly obtain

$$
\left.{\widetilde{y_{a}}}^{\bar{x}^{x_{b}}}={\widetilde{y_{a}}}^{(y} \bar{x}^{x_{b}}\right)={\widetilde{y_{a}}}^{x^{\prime}}=\prod_{n \in x^{\prime-1} a} \widetilde{y_{n}}
$$

where $x^{\prime}=\prod_{m \in b y^{-1}} \overline{x_{m}}$.

Therefore, if we write all above results together, then we get

$$
\left(\overline{1}, \widetilde{y_{a}}\right)\left(\overline{x_{b}}, \tilde{1}\right)=\left(\prod_{m \in b y^{\prime-1}} x_{m}\right)\left(\prod_{n \in x^{\prime-1} a} y_{n}\right),
$$

as required. As a result of all these above findings, we deduce that $\rho$ defines actually an epimorphism $\bar{\rho}$ from the monoid $M$ obtained by relations (2.3), (2.4), (2.5) and (2.6) onto the monoid $A^{\oplus B}{ }_{\delta} \times{ }_{\psi} B^{\oplus A}$.

Now we need to prove that $\rho$ is a monomorphism. Let $w$ be a word representing an element of $M$. By using relations (2.4), (2.5) and (2.6), it is easy to show that there exist words $(b) w$ in $X^{*}(b \in B)$ and $(a) w$ in $Y^{*}(a \in A)$ such that

$$
w=\left(\prod_{b \in B}((b) w)_{b}\right)\left(\prod_{a \in A}((a) w)_{a}\right)
$$

in $M$. (We note that if $z \in X^{*}, t \in Y^{*}$ then $z_{b}$ and $t_{a}$ are the corresponding words in $X_{b}^{*}$ and $Y_{a}^{*}$, respectively). Now, for each $w \in X^{*} \cup Y^{*}, c \in B$ and $d \in A$, we have

$$
(c) \overline{w_{b}}=\left\{\begin{array}{ll}
w, & b=c \\
1, & \text { otherwise }
\end{array} \quad \text { and } \quad(d) \widetilde{w_{a}}=\left\{\begin{array}{ll}
w, & a=d \\
1, & \text { otherwise }
\end{array} .\right.\right.
$$

Hence we get

$$
\begin{gathered}
(c)\left(\prod_{b \in B} \overline{((b) w)_{b}}\right)=\prod_{b \in B}(c) \overline{((b) w)_{b}}=(c) w \\
(d)\left(\prod_{a \in A}\left(\widetilde{(a) w)_{a}}\right)=\prod_{a \in A}(d)\left(\widetilde{(a) w)_{a}}=(d) w\right.\right.
\end{gathered}
$$

for all $c \in B$ and $d \in A$. 
For any two words $u, v$ in $\left(\left(\bigcup_{b \in B} X_{b}\right) \cup\left(\bigcup_{a \in A} Y_{a}\right)\right)^{*}$, we have

$$
\begin{aligned}
(u) \rho=(v) \rho & \Rightarrow \quad\left(\left(\prod_{b \in B}((b) u)_{b}\right)\left(\prod_{a \in A}((a) u)_{a}\right)\right) \rho=\left(\left(\prod_{b \in B}((b) v)_{b}\right)\left(\prod_{a \in A}((a) v)_{a}\right)\right) \rho \\
& \Rightarrow \quad\left(\left(\prod_{b \in B}((b) u)_{b}\right) \rho\left(\prod_{a \in A}((a) u)_{a}\right)\right) \rho=\left(\left(\prod_{b \in B}((b) v)_{b}\right) \rho\left(\prod_{a \in A}((a) v)_{a}\right)\right) \rho \\
& \Rightarrow \quad\left(\prod_{b \in B}\left(\overline{(b) u)_{b}}, \tilde{1}\right)\right)\left(\prod_{a \in A}\left(\overline{1},\left(\widetilde{(a) u)_{a}}\right)\right)=\left(\prod _ { b \in B } ( ( \overline { ( b ) v ) _ { b } } , \tilde { 1 } ) ) \left(\prod_{a \in A}\left(\overline{1},\left(\widetilde{(a) v)_{a}}\right)\right)\right.\right.\right. \\
& \Rightarrow \quad\left(\prod _ { b \in B } \left(\overline{(b) u)_{b}}, \prod_{a \in A}\left(\left(\left(\widetilde{(a) u)_{a}}\right)\right)=\left(\prod_{b \in B}\left(\overline{((b) v)_{b}}, \prod_{a \in A}\left(\widetilde{(a) v)_{a}}\right)\right) .\right.\right.\right.\right.
\end{aligned}
$$

Now from the equality of the first and second components and using equalities (2.7)-(2.8), we deduce that $(c) u=(c) v$ in $A$ (for all $c \in B$ ) and $(d) u=(d) v$ in $B$ (for all $d \in A$ ). Also, relations given in (2.3) imply $u=v$ in the monoid $M$. Therefore $\bar{\rho}$ is injective.

These complete the proof.

Remark 2.3. For $d \in x^{-1} a$ and $e \in b y^{-1}$, since $(d){\widetilde{y_{a}}}^{x}=y$ and $(e)^{y} \overline{x_{b}}=x$, we have seen in the above proof there exist equalities

$$
{ }^{\left(\widetilde{y}^{x}\right)} \overline{x_{b}}={ }^{y} \overline{x_{b}}={ }^{\prime} \overline{x_{b}} \quad \text { and } \quad{\widetilde{y_{a}}}^{\left(y \overline{x b}_{b}\right)}={\widetilde{y_{a}}}^{x}={\widetilde{y_{a}}}^{x^{\prime}} .
$$

Therefore, by omitting the bar and tilde signs, another version of the relation given in (2.6) can be stated as

$$
y_{a} x_{b}=\left(\prod_{n \in x^{-1} a} y_{n}\right)_{x_{b} y_{a}}\left(\prod_{m \in b y^{-1}} x_{m}\right) .
$$

We have the following consequence of Theorem 2.2.

Corollary 2.4. Let $A$ and $B$ be monoids with the conditions given in Theorem 2.2 hold. Then the standard presentation for $A^{\oplus B}{ }_{\delta} \times{ }_{\psi} B^{\oplus A}$ is given by

$$
\begin{gathered}
{\left[X_{b}, Y_{a} ; \quad R_{b}, S_{a} \quad(a \in A, b \in B),\right.} \\
x_{b} x_{e}^{\prime}=x_{e}^{\prime} x_{b} \quad\left(x, x^{\prime} \in X, b, e \in B, b \neq e\right), \\
y_{a} y_{s}^{\prime}=y_{s}^{\prime} y_{a} \quad\left(y, y^{\prime} \in Y, a, s \in A, a \neq s\right), \\
y_{a} x_{b}=\left(\prod_{n \in x^{-1} a} y_{n}\right)_{x_{b} y_{a}}\left(\prod_{m \in b y^{-1}} x_{m}\right) .
\end{gathered}
$$

At the rest of this section, as a special case of Theorem 2.2 (and also Corollary 2.4), we will only consider the generalized Zappa product $A^{\oplus B}{ }_{\delta} \times_{\psi} B$ for defining a presentation on it.

For an arbitrary monoid $A$ with a presentation $[X ; R]$ and an arbitrary monoid $B$ with a presentation $[Y ; S]$, let us consider

$$
\begin{aligned}
& \delta \quad: \quad B \rightarrow \mathcal{T}\left(A^{\oplus B}\right) \quad \text { and } \quad \psi \quad: \quad A^{\oplus B} \rightarrow \mathcal{T}(B) \\
& b \mapsto(g) \delta_{b}={ }^{b} g \quad g \mapsto(b) \psi_{g}=b^{g}
\end{aligned}
$$

such that $(x)^{b} g=(x b) g$ for $x \in B$ and $b^{g}=b^{\left(b^{\prime} g\right)}$ for $b^{\prime} \in B$. Then the generalized Zappa product $A^{\oplus B}{ }_{\delta} \times_{\psi} B$ is defined on the set $A^{\oplus B} \times B$ with a multiplication $(f, b)\left(g, b^{\prime}\right)=$ $\left(f^{b} g, b^{g} b^{\prime}\right)$. 
Theorem 2.5. A presentation for $A^{\oplus B}{ }_{\delta} \times{ }_{\psi} B$ is defined by

$$
\left[X_{b}, Y ; R_{b}, S, x_{b} x_{e}^{\prime}=x_{e}^{\prime} x_{b}, y x_{b}=\left(\prod_{m \in b y^{-1}} x_{m}\right) y^{\left(\prod_{m \in b y^{-1}} x_{m}\right)}\right],
$$

where $x, x^{\prime} \in X, y \in Y, b, e \in B, b \neq e$.

Proof. Let us consider the presentation given in Corollary 2.4. Since we have just one copy of $B$ in the product $A^{\oplus B}{ }_{\delta} \times_{\psi} B$, we must have $Y$ instead of $Y_{a}$ in the generating set and also $S$ instead of $S_{a}$ in the relators set of the requiring presentation. Moreover, by the same reason, the relator $y_{a} y_{s}^{\prime}=y_{s}^{\prime} y_{a}\left(y, y^{\prime} \in Y, a, s \in A, a \neq s\right)$ will be disappeared.

For the last relator, again let us consider the multiplication $(\overline{1}, y)\left(\overline{x_{b}}, 1_{B}\right)=\left({ }^{y} \overline{x_{b}}, y^{\overline{x_{b}}}\right)$, where $x \in X, y \in Y$ and $b \in B$. Recall that, in the proof of Theorem 2.2, we obtained the equation

$$
{ }^{y} \overline{x_{b}}=\prod_{m \in b y^{-1}} \overline{x_{m}} .
$$

Hence, by considering both (2.6) and (2.9) with the fact that there exists a single $B$ in the product $A^{\oplus B}{ }_{\delta} \times_{\psi} B$, we obtain

$$
(\overline{1}, y)\left(\overline{x_{b}}, 1_{B}\right)=\left(\prod_{m \in b y^{-1}} x_{m}\right) y^{\left(\prod_{m \in b y^{-1}} x_{m}\right)}
$$

as required.

Notice that presentation in (2.10) is a generalization of the presentation given in (1.4) since it presents a product having mutual actions.

As a consequence of Theorem 2.5, we can get a much nicer presentation in the case of $B$ is a group which is actually a generalization of the presentation defined in [18, Corollary $2.3]$.

Corollary 2.6. Assume that $A$ is a monoid but $B$ is a group. Now consider their monoid presentations $[X ; R]$ and $[Y ; S]$, respectively. Thus $A^{\oplus B}{ }_{\delta} \times{ }_{\psi} B$ has a presentation

$$
\left[X, Y ; R, S, x\left(b^{-1} x^{\prime} b^{x^{\prime \prime}}\right)=\left(b^{-1} x^{\prime} b^{x^{\prime \prime}}\right) x\right],
$$

where $x, x^{\prime}, x^{\prime \prime} \in X, b \in B$.

Proof. Recall from (1.2), for any $a \in A$ and $b \in B$, the action satisfies $b a={ }^{b} a b^{a}$. So, for $x_{b} \in A^{\oplus B}$ and $b \in B$, we get

$$
b x_{b}={ }^{b} x_{b} b^{x_{b}} .
$$

Now, by replacing $b$ instead of $y$ in equations ${ }^{y} \overline{x_{b}}=\prod_{m \in b y^{-1}} \overline{x_{m}}$ and $y^{\overline{x_{b}}}=y^{\left({ }^{y} \overline{x_{b}}\right)}$, where $m \in B$, which are obtained in Theorems 2.2 and 2.5 and also by writing those new equations in (2.11), we obtain the relation

$$
\prod_{b x_{b}=x_{1_{B}} b^{m \in b y^{-1}}} x_{m}
$$

in $A^{\oplus B}{ }_{\delta} \times{ }_{\psi} B$. For just simplicity, if we write $x^{\prime}$ instead of $x_{1_{B}}$ and $x^{\prime \prime}$ instead of $\prod_{m \in b y^{-1}} x_{m}$, then this above last relation becomes

$$
x_{b}=b^{-1} x^{\prime} b^{x^{\prime \prime}} .
$$

Further, by using (2.12), if we eliminate the element $x_{b}$ (where $x \in X, b \in B-\left\{1_{B}\right\}$ ) from the relations in presentation (2.10), the last relator of this presentation becomes trivial while the relations $R_{b}$ and $x_{b} x_{e}^{\prime}=x_{e}^{\prime} x_{b}$ are actually consequences of the relations $R$ and 
$x\left(b^{-1} x^{\prime} b^{x^{\prime \prime}}\right)=\left(b^{-1} x^{\prime} b^{x^{\prime \prime}}\right) x$, respectively, in the meaning of Tietze transformations, where $x, x^{\prime}, x^{\prime \prime} \in X, b \in B$.

Hence this completes the proof.

By taking into account both $A$ and $B$ as any groups, Corollary 2.6 can be expressed as in the following.

Corollary 2.7. Assume that both $A$ and $B$ are groups with their monoid presentations $[X ; R]$ and $[Y ; S]$, respectively. Hence the presentation

$$
\left[X, Y ; R, S, a\left(b^{-1} a^{\prime} b^{a^{\prime \prime}}\right)=\left(b^{-1} a^{\prime} b^{a^{\prime \prime}}\right) a \quad\left(b \in B, a, a^{\prime}, a^{\prime \prime} \in A\right)\right]
$$

defines $A^{\oplus B}{ }_{\delta} \times_{\psi} B$.

Proof. As in the proof of Corollary 2.6, for $a \in A$ and $b \in B$, we can easily see that

$$
\prod_{a_{b}=b^{-1} a_{1_{B}} b^{m \in b y^{-1}}} a_{m}
$$

holds in $A^{\oplus B}{ }_{\delta} \times_{\psi} B$. For simplicity, let us replace $a_{1_{B}}$ by $a^{\prime}$ and $\prod_{m \in b y^{-1}} a_{m}$ by $a^{\prime \prime}$. Then the above equality becomes $a_{b}=b^{-1} a^{\prime} b^{a^{\prime \prime}}$. Therefore, by replacing $a_{b}$ in presentation (2.10), we obtain the required presentation given in the statement of corollary.

\section{Some applications}

By considering the presentation defined in Theorem 2.5 for $A^{\oplus B}{ }_{\delta} \times_{\psi} B$, we will give some examples while $A$ and $B$ are taken as some special monoids.

\subsection{Finite case}

In this section we will study on finite cyclic monoids (cf. [19]). In fact some examples and applications over other extensions for these monoids have been investigated, for instance, in $[3,4,15]$.

Suppose that $A=\left[x ; x^{k}=x^{l}(k>l)\right]$ and $B=\left[y ; y^{s}=y^{t}(s>t)\right]$ are finite cyclic monoids, and consider $\delta$ and $\psi$ as given in Theorem 2.5. We then have the following result.

Corollary 3.1. Let $A$ and $B$ be finite cyclic monoids as in above. Then

$$
\begin{aligned}
{\left[x^{(0)}, x^{(1)}, \cdots, x^{(s-1)}, y \quad ;\right.} & y^{s}=y^{t}, x^{(i)} x^{(j)}=x^{(j)} x^{(i)} \quad(0 \leq i<j \leq s-1), \\
& x^{(i)^{k}}=x^{(i)^{l}} \quad(0 \leq i \leq s-1) \\
& y x^{(i)}=x^{(i-1)} y^{x^{(i-1)}} \quad(1 \leq i \leq s-1) \\
& \left.y x^{(t)}=x^{(s-1)} y^{x^{(s-1)}}\right]
\end{aligned}
$$

is a presentation for the product $A^{\oplus B}{ }_{\delta} \times_{\psi} B$.

Proof. By considering $A$ and $B$ are finite cyclic monoids, we just need to convert presentation (2.10) in Theorem 2.5. For all $y^{i} \in B$, let us label each $x_{y^{i}}$ by $x^{(i)}$, where $0 \leq i \leq s-1$, for simplicity. Therefore the set of the generators for the monoid $A^{\oplus B}{ }_{\delta} \times_{\psi} B$ is $\left\{x^{(i)}, y\right\}$. Further, since $A^{\oplus B}$ is a direct product, we must have $x^{(i)} x^{(j)}=x^{(j)} x^{(i)}(0 \leq i<j \leq s-1)$ and $x^{(i)^{k}}=x^{(i)^{l}}$ as relations in our presentation.

Now let us consider the relator

$$
y x_{b}=\left(\prod_{m \in b y^{-1}} x_{m}\right) y^{\left(\prod_{m \in b y^{-1}} x_{m}\right)}
$$


in presentation (2.10). In this relator, by taking $1, y, y^{2}, \cdots, y^{s-1}$ instead of each $b \in B$ and replacing each $x_{b}$ by related $x^{(i)}$ where $0<i \leq s-1$, we obtain the relator $y x^{(i)}=$ $x^{(i-1)} y^{x^{(i-1)}}$. Moreover, for the monoid $B$, since we have $y^{s}=y^{t}$ as a relator, we can write this relator as $y^{t}=y^{s-1} y$ which implies that, for $b=y^{t}$ and $m=y^{s-1}, y x^{(t)}=x^{(s-1)} y^{x^{(s-1)}}$ by keeping same idea as in the previous sentence.

Hence this completes the proof.

We can also give the following application which is a consequence of Corollary 2.6.

Corollary 3.2. Let $A$ be a finite monoid (not necessarily cyclic) and let $B$ be a cyclic group of order s. If $\mathcal{P}_{A}=[X ; R]$ and $\mathcal{P}_{B}=\left[y ; y^{s}=y^{t}(s>t)\right]$ are their monoid presentations, respectively, then the presentation

$$
\left[X, y ; R, y^{s}=y^{t}, x\left(y^{-i} x^{\prime}\left(y^{i}\right)^{x^{\prime}}\right)=\left(y^{-i} x^{\prime}\left(y^{i}\right)^{x^{\prime}}\right) x \quad\left(x, x^{\prime} \in X, 0<i \leq(s-t)-1\right)\right]
$$

defines the product $A^{\oplus B}{ }_{\delta} \times_{\psi} B$.

Proof. From Corollary 2.6, we have the relations $b x_{b}=x_{1_{B}} b^{x_{1}}$, for $b \in B, x \in X$. If we take $1, y, y^{2}, \cdots, y^{(s-t)-1}$ instead of for each $b$, we obtain $x^{(i)}=y^{-i} x^{(0)}\left(y^{i}\right)^{x^{(0)}}$ where $0<i \leq(s-t)-1$. Also let us replace $x^{\prime}$ by $x^{(0)}$. Thus we have $x^{(i)}=y^{-i} x^{\prime}\left(y^{i}\right)^{x^{\prime}}$. Hence this completes the proof.

\subsection{Infinite case}

In this subcase, let $A$ be the free Abelian monoid rank 2 and let $B$ be the finite cyclic monoid. As a consequence of Theorem 2.5, we have the following result which can be proved quite similarly as in Corollary 3.1 .

Corollary 3.3. Let $\mathcal{P}_{A}=\left[x_{1}, x_{2} ; x_{1} x_{2}=x_{2} x_{1}\right]$ and $\mathcal{P}_{B}=\left[y ; y^{s}=y^{t}(s>t)\right]$ be monoid presentations for the above monoids $A$ and $B$. Therefore, the monoid $A^{\oplus B}{ }_{\delta} \times_{\psi} B$ has a presentation with generators

$$
x_{1}^{(0)}, x_{1}^{(1)}, \cdots, x_{1}^{(s-1)}, x_{2}^{(0)}, x_{2}^{(1)}, \cdots, x_{2}^{(s-1)}, y
$$

and relators

$$
\begin{aligned}
y^{s}=y^{t}, x_{i}^{(m)} x_{j}^{(n)}=x_{j}^{(n)} x_{i}^{(m)} & (i, j \in\{1,2\}, \quad 0 \leq m, n \leq s-1), \\
y x_{1}^{(m)}=x_{1}^{(m-1)} y^{x_{1}^{(m-1)}} & (0<m \leq s-1), \\
y x_{2}^{(n)}=x_{2}^{(n-1)} y^{x_{2}^{(n-1)}} & (0<n \leq s-1), \\
y x_{1}^{(t)}=x_{1}^{(s-1)} y^{x_{1}^{(s-1)}}, & y x_{2}^{(t)}=x_{2}^{(s-1)} y^{x^{(s-1)}} .
\end{aligned}
$$

We note that Corollary 3.3 can be easily generalized for an arbitrary free abelian monoid $A$ with rank greater than 2 .

On the other hand another infinite case application of Theorem 2.5 is the following:

Let $A$ be the free monoid with a presentation $\mathcal{P}_{A}=[x ;]$ and let $B$ be the monoid $\mathbb{Z}_{s} \times \mathbb{Z}_{m}$ with a presentation

$$
\mathcal{P}_{B}=\left[y_{1}, y_{2} ; y_{1}^{s}=y_{1}^{t}, y_{2}^{m}=y_{2}^{n}(s>t, m>n), y_{1} y_{2}=y_{2} y_{1}\right] .
$$

For a representive element $y_{1}^{i} y_{2}^{j}$ in the monoid $B$, let us label $x_{y_{1}^{i} y_{2}^{j}}$ by $x^{(i, j)}$ where $0 \leq i \leq$ $s-1,0 \leq j \leq m-1$. Then, for each element in $B$, we have a generating set $\left\{x^{(i, j)}, y_{1}, y_{2}\right\}$ for the monoid $A^{\oplus B}{ }_{\delta} \times_{\psi} B$. Therefore, by suitable changes in presentation (2.10), we obtain the following result. 
Corollary 3.4. Let $A$ and $B$ be as above. Then

$$
\begin{aligned}
{\left[x^{(i, j)}, y_{1}, y_{2} \quad ; \quad\right.} & y_{1}^{s}=y_{1}^{t}, y_{2}^{m}=y_{2}^{n}(s>t, m>n), y_{1} y_{2}=y_{2} y_{1}, \\
& x^{(i, j)} x^{(l, k)}=x^{(l, k)} x^{(i, j)} \quad(0 \leq i \leq s-1,0 \leq j \leq m-1,(i, j)<(l, k)), \\
& y_{1} x^{(i, j)}=x^{(i-1, j)} y_{1}^{x^{(i-1, j)}} \quad(1 \leq i \leq s-1,0 \leq j \leq m-1), \\
& y_{2} x^{(i, j)}=x^{(i, j-1)} y_{2}^{x^{(i, j-1)}} \quad(0 \leq i \leq s-1,1 \leq j \leq m-1), \\
& y_{1} x^{(t, j)}=x^{(s-1, j)} y_{1}^{x^{(s-1, j)}} \quad(0 \leq j \leq m-1), \\
& y_{2} x^{(i, n)}=x^{(i, m-1)} y_{2}^{x^{(i, m-1)}} \quad(0 \leq i \leq s-1)
\end{aligned}
$$

is a presentation for $A^{\oplus B}{ }_{\delta} \times_{\psi} B$.

\section{Conclusions and future problems}

In this paper, we first introduced a new monoid $A^{\oplus B}{ }_{\delta} \times{ }_{\psi} B^{\oplus A}$ under the name of $a$ higher version of Zappa products or generalized Zappa products of the monoid $A^{\oplus B}$ by the monoid $B^{\oplus A}$ which is obtained by a combination of Zappa and wreath products. Then we defined a presentation on this new Theorem 2.2. After that, by taking $A$ and $B$ as finite (or infinite) monoid examples and also taking them as groups with their monoid presentations, we presented some consequences of Theorem 2.2.

It is clear that to define a presentation on an algebraic structure is an important tool in geometric group theory since this implies new studying areas over this structure. So, by considering the presentation defined in Theorem 2.2 or the presentations defined in corollaries of Theorem 2.2, one may study Gröbner-Shirshov bases (see, for instance, $[12,21]$ ) over these presentations since the normal forms obtained by Gröbner-Shirshov bases implies the solvability of word problems ([11]). Furthermore the existence of other decision problems, specially the isomorphism problem, over the monoid $A^{\oplus B}{ }_{\delta} \times{ }_{\psi} B^{\oplus A}$ can be studied for a future project. Additionally, with the help of Theorem 2.2, the subjects Green's relations, periodicity and local finiteness may also be studied on $A^{\oplus B}{ }_{\delta} \times{ }_{\psi} B^{\oplus A}$.

Another future research on $A^{\oplus B}{ }_{\delta} \times{ }_{\psi} B^{\oplus A}$ would be the adaptation of the results presented in [7] and [8], that is, to investigate whether there exists a bijective correspondence between formations of the monoid $A^{\oplus B}{ }_{\delta} \times{ }_{\psi} B^{\oplus A}$ with formations of languages.

Acknowledgment. The authors would like to thank referees for their valuable suggestions and comments. This work was funded by the Deanship of Scientific Research (DSR), King Abdulaziz University, Jeddah, under grant No (G. 1711-130-1440). The authors, therefore, acknowledge with thanks DSR technical and financial support.

\section{References}

[1] F. Ates and A.S. Cevik, Knit products of finite cyclic groups and their applications, Rend. Sem. Mat. Univ. Padova, 121, 1-12, 2009.

[2] H. Ayik, C.M. Campbell, J.J. O'Connor and N. Ruskuc, On the efficiency of wreath products of groups, Groups-Korea 98, in: Proceedings of the International Conference held at Pusan National University, Pusan, Korea, August 10-16, 1998, Walter de Gruyter, 39-51, 2000.

[3] H. Ayik, C.M. Campbell, J.J. O'Connor and N. Ruskuc, Minimal presentations and efficiency of semigroups, Semigroup Forum, 60, 231-242, 2000.

[4] H. Ayik, F. Kuyucu and B. Vatansever, On semigroup presentations and efficiency, Semigroup Forum, 65, 329-335, 2002. 
[5] A. Ballester-Bolinches, E. Cosme-Llopez and R. Esteban-Romero, Group extensions and graphs, Expo. Math. 34 (3), 327-334, 2016.

[6] A. Ballester-Bolinches, R. Esteban-Romero and M. Asaad, Products of Finite Groups, de Gruyter Exp. Math. 53, Walter de Gruyter, 2010.

[7] A. Ballester-Bolinches, J.E. Pin and X. Soler-Escriva, Formations of finite monoids and formal languages: Eilenberg's variety theorem revisited, Forum Math. 26 (6), 1737-1761, 2014.

[8] A. Ballester-Bolinches, E. Cosme-Llopez, R. Esteban-Romero and J.J.M.M. Rutten, Formations of monoids, congruences, and formal languages, Sci. Ann. Comput. Sci. 25 (2), 171-209, 2015.

[9] A. Ballester-Bolinches, L.M. Ezquerro, A.A. Heliel and M.M. Al-Shomrani, Some results on products of finite groups, Bull. Malays. Math. Sci. Soc. 40 (3), 1341-1351, 2017.

[10] G. Baumslag, Wreath products and finitely presented groups, Math. Z. 75, 22-28, 1961.

[11] L.A. Bokut, Unsolvability of the word problem, and subalgebras of finitely presented Lie algebras, Izv. Akad. Nauk. SSSR Ser. Math. 36, 1173-1219, 1972.

[12] L.A. Bokut, Y. Chen and X. Zhao, Gröbner-Shirshov bases for free inverse semigroups, Internat. J. Algebra Comput. 19 (2), 129-143, 2009.

[13] M.G. Brin, On the Zappa-Szép product, Comm. Algebra 33, 393-424, 2005.

[14] A.S. Cevik, The efficiency of standard wreath product, Proc. Edinburgh Math. Soc. 43 (2), 415-423, 2000.

[15] A.S. Cevik, Minimal but inefficient presentations of the semi-direct product of some monoids, Semigroup Forum, 66 (1), 1-17, 2003.

[16] N.D. Gilbert and S. Wazzan, Zappa-Szép products of bands and groups, Semigroup Forum, 77, 438-455, 2008.

[17] A.A. Heliel, A. Ballester-Bolinches, R. Esteban-Romero and M.O. Almestady, Zpermutable subgroups of finite groups, Monat. Math. 179 (4), 523-534, 2016.

[18] J.M. Howie and N. Ruskuc, Constructions and presentations for monoids, Comm. Algebra, 22 (15), 6209-6224, 1994.

[19] J.M. Howie, Fundamentals of Semigroup Theory, London Math. Soc. Monographs, Oxford University Press, 1995.

[20] D.L. Johnson, Presentation of Groups, London Math. Soc. Lecture Note Series 15, Cambridge University Press, 1990.

[21] C. Kocapinar, E.G. Karpuz, F. Ates and A.S. Cevik, Gröbner-Shirshov bases of the generalized Bruck-Reilly *-extension, Algebra Colloq. 19, 813-820, 2012.

[22] M. Kunze, Zappa products, Acta Math. Hung. 41, 225-239, 1983.

[23] T.G. Lavers, Presentations of general products of monoids, J. Algebra 204, 733-741, 1998.

[24] S. MacLane, Homology, Classics in Mathematics, Springer Verlag, 1975.

[25] J.D.P. Meldrum, Wreath Products of Groups and Semigroups, Monographs and Surveys in Pure and Applied Mathematics (Book 74), Chapman and Hall/CRC; First Edition, 1995.

[26] P.W. Michor, Knit products of graded Lie algebras and groups, Rend. Circ. Mat. Palermo (2), 22, 171-175, 1989.

[27] J. Szép, On the structure of groups which can be represented as the product of two subgroups, Acta Sci. Math. Szeged, 12, 57-61, 1950.

[28] G. Zappa, Sulla costruzione dei gruppi prodotto di due sottogruppi permutabili tra loro, in: Atti Secondo Congresso Un. Ital., Bologna 1940. Edizioni Rome: Cremonense, 119-125, 1942. 